\title{
N-Methyl-o-Aspartate Receptor Antibodies in Herpes Simplex Encephalitis
}

\author{
Harald Prüss, M.D. ${ }^{1}$, Carsten Finke, M.D. ${ }^{1}$, Markus Höltje, Ph.D. ${ }^{2}$, Joerg Hofmann, M.D. ${ }^{3}$, \\ Christine Klingbeil ${ }^{4}$, Christian Probst, Ph.D. ${ }^{4}$, Kathrin Borowski ${ }^{4}$, Gudrun Ahnert-Hilger, \\ Ph.D. ${ }^{2}$, Lutz Harms, M.D. ${ }^{1}$, Jan M. Schwab, M.D., Ph.D. ${ }^{1}$, Christoph J. Ploner, M.D. ${ }^{1}$, Lars \\ Komorowski, Ph.D. ${ }^{4}$, Winfried Stoecker, M.D. ${ }^{4}$, Josep Dalmau, M.D., Ph.D. ${ }^{5,6}$, and Klaus- \\ Peter Wandinger, M.D. ${ }^{4,7}$ \\ ${ }^{1}$ Department of Neurology, Charité University Medicine Berlin, Berlin, Germany \\ ${ }^{2}$ Institute for Integrative Neuroanatomy, Charité University Medicine Berlin, Berlin, Germany \\ ${ }^{3}$ Institute of Medical Virology, Helmut-Ruska-Haus, Charité University Medicine Berlin, and Labor \\ Berlin Charité-Vivantes GmbH, Berlin, Germany \\ ${ }^{4}$ Institute for Experimental Immunology, affiliated with Euroimmun, Lübeck, Germany \\ ${ }^{5}$ Catalan Institution for Research and Advanced Studies (ICREA) at Institution of Biomedical \\ Research August Pi i Sunyer, Service of Neurology, Hospital Clinic, University of Barcelona, \\ Barcelona, Spain \\ ${ }^{6}$ Department of Neurology, University of Pennsylvania, Philadelphia, PA \\ ${ }^{7}$ Institute for Neuroimmunology and Clinical Multiple Sclerosis Research, Center for Molecular \\ Neurobiology Hamburg, University Medical Center Eppendorf, Hamburg, Germany
}

\section{Abstract}

Objective-To determine the presence and kinetics of antibodies against synaptic proteins in patients with herpes simplex virus encephalitis (HSE).

Methods-Retrospective analysis of 44 patients with polymerase chain reaction-proven HSE for the presence of a large panel of onconeuronal and synaptic receptor antibodies. The effect of patients' serum was studied in cultures of primary mouse hippocampal neurons.

\begin{abstract}
Results-N-Methyl-D-aspartate receptor (NMDAR) antibodies of the immunoglobulin (Ig) subtypes $\operatorname{Ig} \mathrm{A}, \operatorname{IgG}$, or IgM were detected in 13 of 44 patients (30\%) in the course of HSE, suggesting secondary autoimmune mechanisms. NMDAR antibodies were often present at hospital admission, but in some patients developed after the first week of HSE. Antibody-positive sera resulted in downregulation of synaptic marker proteins in hippocampal neurons.

Interpretation-Some patients with HSE develop IgA, IgG, or IgM autoantibodies against NMDAR. Sera from these patients alter the density of neuronal synaptic markers, suggesting a potential pathogenic disease-modifying effect. These findings have implications for the understanding of autoimmunity in infectious diseases, and prospective studies should reveal
\end{abstract}

Address correspondence to Dr Prüss, Department of Neurology and Experimental Neurology, Charité University Medicine Berlin, Charitéplatz 1, 10117 Berlin, Germany. harald.pruess@ charite.de.

H.P. and C.F. contributed equally to the work.

Potential Conflicts of Interest

C.K.: employment, Euroimmun. C.P.: employment, stock/ stock options, Euroimmun. K.B.: employment, Euroimmun. L.K.: employment, Euroimmun. W.S.: board membership, employment, stock/stock options, Euroimmun. J.D.: grants/grants pending, Euroimmun, NIH/ NCI; patents, Athena Diagnostics, Euroimmun. K.-P.W.: employment, stock/stock options, Euroimmun 
whether the subgroup of patients with HSE and NMDAR antibodies may benefit from immunotherapy.

Herpes simplex encephalitis (HSE) is the most frequent fatal encephalitis in Western countries. ${ }^{1,2}$ Despite its substantially improved prognosis since the advent of selective antiviral therapy with acyclovir, about $35 \%$ of patients still suffer an unfavorable outcome, with severe neurological residual symptoms or even death. ${ }^{3}$ However, in patients with HSE, not all symptoms result from direct virus invasion and neuronal cell lysis. The observation of a more severe disease course in immuno-competent as compared to immunocompromised patients suggests a role for secondary autoimmune mechanisms in the pathogenesis of HSE. ${ }^{4}$ This hypothesis is in line with studies demonstrating a beneficial effect on the outcome when combining acyclovir with corticosteroids. ${ }^{5,6}$ Additionally, direct viral cytotoxicity is probably not the major pathogenic mechanism in relapses of HSE. ${ }^{7,8}$

During clinical workup of encephalitis patients, we identified an HSE case that had hightiter immunoglobulin (Ig)A antibodies against N-methyl-D-aspartate receptors (NMDARs), raising the question of whether some symptoms in HSE might be related to secondary immunological phenomena, such as generation of antibodies against neuronal cell surface antigens. These could include prolonged symptoms after acyclovir treatment, the presence of unusual clinical presentations, and the beneficial effect of steroids in some patients. To get an unbiased estimation of the true prevalence of antibodies against a wide range of NMDARs (different subtypes and epitopes) and other synaptic proteins in HSE, we performed a blinded retrospective study analyzing a large archived cohort of consecutive serum and cerebrospinal fluid (CSF) samples from patients with a definite diagnosis of HSE.

\section{Patients and Methods}

\section{Patients}

Archived serum and CSF samples from 44 consecutive patients (27 males; mean age, $52.7 \pm$ 16.6 years; range, 12-83 years) with polymerase chain reaction (PCR)-proven HSE (>500 herpes simplex virus [HSV] DNA copies/ml, 80\% >2,000 copies/ ml), seen between 2005 and 2012 at the Charite University Hospital, were retrospectively analyzed for onconeuronal and synaptic receptor antibodies. All patients fulfilled the clinical criteria of $\mathrm{HSE}^{9}$ in accordance with the German Society of Neurology guidelines, had compatible laboratory and imaging findings (eg, T2w lesions of the medial temporal lobes), and received intravenous acyclovir treatment $(3 \times 10-15 \mathrm{mg} / \mathrm{kg})$ for at least 14 days. CSF parameters (white blood cell count, CSF protein, oligoclonal bands) were recorded during routine clinical testing in the CSF laboratory of the Charité University Hospital. Patients with PCRproven enterovirus encephalitis $(n=10)$ and varicella zoster virus $(\mathrm{VZV})$ encephalitis $(\mathrm{n}=$ 10) served as controls. Retrospective analyses were approved by the Charite University Hospital Institutional Review Board, and written informed consent for material storage was obtained from patients or their representatives.

\section{Detection of NMDAR Antibodies and Intrathecal Antibody Synthesis}

Testing for NMDAR antibodies was performed by recombinant immunofluorescence. ${ }^{10}$ Briefly, plasmids encoding the NMDA type glutamate receptor (using NR1a subunit homodimers and equimolar NR1a/NR2b heterodimers in parallel experiments $)^{11}$ were transfected into HEK293 cells. Transfected cells were grown on cover slides, followed by acetone fixation. Coated cover glasses were cut into millimeter-sized fragments (biochips) and used side by side with cells transfected with an empty plasmid in a mosaic that additionally contained HEK 293 cells transfected with glutamate receptor (type AMPA; GluR1/ GluR2), $\gamma$-aminobutyric acid (GABA) receptor (B1), LGI1, CASPR2, and GAD65 
and frozen sections of rat hippocampus and cerebellum. Slides were incubated with blinded patient samples at a starting dilution of 1:10 (serum) or undiluted (CSF). After 30 minutes at room temperature, slides were washed with phosphate-buffered saline (PBS)-Tween for $>5$ minutes. Bound antibodies were labeled using individual stainings with fluoresceinconjugated goat-anti-human IgG (dilution 1:800), IgA (1:350), or IgM (1:500) antibodies (purchased from DiaMed, Canton, $\mathrm{OH}$ ) for 30 minutes. Coded samples were classified positive or negative by 2 independent assessors based on intensity of immunofluorescence of transfected cells in direct comparison with control-transfected cells and control samples. Typical findings are shown in Figure 1. Classical paraneoplastic antibodies (ie, anti-Hu, Yo, -Ri, -Ma, -CV2, -amphiphysin) were determined based on the characteristic tissue pattern in indirect immunofluorescence and by means of a monospecific, recombinant line immunoblot assay (Euroimmun, Lübeck, Germany).

Quantification of antigen-specific intrathecal antibody synthesis was determined as described in detail elsewhere. ${ }^{12}$ Briefly, NMDAR-specific antibody index values were calculated as the ratio between the CSF/serum quotient for NMDAR antibodies and the $\mathrm{CSF} /$ serum quotient for total immunoglobulin of the relevant class. Values $>4$ were considered as evidence of intrathecal NMDAR-specific antibody synthesis. Antigenindependent intrathecal synthesis, reflecting autoimmunity in the central nervous system (CNS), was calculated from total IgG, IgM, or IgA antibody index using the Reibergram calculation. $^{12}$

\section{Primary Hippocampal Neurons}

Cultures of dissected mice hippocampal neurons were obtained as previously reported. ${ }^{13}$ In brief, hippocampi at embryonic day 16 were dissociated in minimum essential medium supplemented with $10 \%$ fetal calf serum, 100IE insulin/l, 0.5mM glutamine, $100 \mathrm{U} / \mathrm{ml}$ penicillin/streptomycin, 44mM glucose, and 10mM N-2-hydroxyethylpiperazine- $\mathrm{N}^{\prime}$-2ethanesulfonic acid (HEPES). Following centrifugation, cells were resuspended (serum-free neurobasal medium supplemented with B27, 0.5mM glutamine, 100U/ml penicillin/ streptomycin and $25 \mu \mathrm{M}$ glutamate), and $8 \times 10^{4}$ cells/well were plated in 24 -well dishes on cover slips precoated with poly-L-lysine/collagen (all ingredients from Gibco/BRL, Eggenstein, Germany).

\section{Western Blot}

At day 10, in vitro hippocampal neurons were harvested and resuspended with $\mathrm{PBS}: \mathrm{H}_{2} \mathrm{O}$ 1:9 (with protease inhibitors and 10mM HEPES, $\mathrm{pH}$ 7.4) for hypo-osmotic cell lysis. Following homogenization with a glass/Teflon homogenizer, a postnuclear supernatant was obtained by 10 -minute centrifugation at 5,000rpm. To obtain the membrane fraction, the supernatant was spun down for 30 minutes at 80,000rpm. Membrane pellets were resolved in Laemmli buffer, separated by sodium dodecyl sulfate-polyacrylamide gel electrophoresis, and transferred to nitrocellulose membranes. The NR1 subunit of the NMDAR was detected with monoclonal antibodies (1:5,000; Synaptic Systems, Göttingen, Germany) using enhanced chemiluminescence. Actin polyclonal antiserum was used as control (1:2,000; Sigma, Deisenhofen, Germany).

\section{Immunocytochemistry}

At day 7, in vitro cultured neurons were incubated with patient or control serum (1:100) for 3 days. Cells were fixed with $4 \%$ formaldehyde for 15 minutes and permeabilized for 30 minutes at room temperature using $0.3 \%$ Triton- $\times 100 / \mathrm{PBS}$. Cultures were stained with primary anti-synapsin (rabbit polyclonal 1:500; Synaptic Systems) antibodies overnight at $4^{\circ} \mathrm{C}$. After washing in PBS, Alexa 594-conjugated secondary antibody was applied for 1 hour at room temperature (Molecular Probes, Eugene, OR). For quantification of synapsin 
immunosignals, 10 to 15 view fields at $\times 40$ magnification $(350 \times 262 \mu \mathrm{m})$ were evaluated per condition in each individual experiment. Images were converted to grayscale by Photoshop software (Adobe Systems, San Jose, CA). Brightness and contrast were uniformly adjusted so that the brightest spots exhibited average grayscale values of 120 to 130. Images were analyzed by Scion Image software (Scion, now Bio-Soft Net). After thresholding, particle counts were analyzed. Individual synaptic spots ranged between 5 and 20 pixels.

\section{Results \\ Detection of NMDAR Autoantibodies}

NMDAR antibodies were detected in serum or CSF of 13 of $44(30 \%)$ patients with HSE (Tables 1 and 2). NMDAR antibodies of the $\operatorname{IgA}$ class were present in $9, \operatorname{IgG}$ in 5, and $\operatorname{IgM}$ in 9 patients. Intrathecal antibody synthesis as demonstrated by a NMDAR-specific antibody index $>4$ or the presence of NMDAR antibodies in the CSF only was observed in 6 patients. Intrathecal production of IgG antibodies directed against the NR1a subunit of the receptor, a typical feature observed in NMDAR encephalitis, was demonstrated in 3 patients (patients 8 , 9, and 11). In 2 patients (patients 2 and 5), NMDAR antibodies were only reactive with transfected cells expressing both the NR1a and NR2b subunits (not the NR1a subunit alone), suggesting that they were directed against a different epitope of the NMDAR, thus showing a broader antibody repertoire than in classical anti-NMDAR encephalitis. This concept is supported by the finding of additional antibodies reactive with neuropil in 1 of the patients (patient 9, not shown). No onconeuronal antibodies or antibodies against other specific synaptic proteins were detected. The clinical and demographic data of NMDAR antibodypositive patients are given in Table 1. NMDAR antibodies were detected in none of 75 healthy older individuals and in only 1 of 29 patients with dementia. ${ }^{14}$

When including only positive samples using more stringent starting dilutions of 1:10 for CSF and 1:100 for serum (as performed in other laboratories), still 11 of 44 (25\%) HSE patients had NMDAR antibodies in CSF and/or serum (7 IgA, $5 \mathrm{IgG}, 4 \mathrm{IgM})$. In the remaining samples (CSF $<1: 10$, serum $<1: 100)$, more concentrated material might potentially increase nonspecific binding, although lower titers were shown in longitudinal studies, ${ }^{10,15}$ and these samples still downregulate NMDAR in hippocampal neurons (Fig 2).

The binding of IgG and IgA antibodies to transfected cells and brain sections has been shown in previous publications ${ }^{11,14}$; however, the presence of IgM antibodies has not been described. We now demonstrate that NMDAR-transfected HEK cells are similarly reactive with antibodies of the IgM class (see Fig 1A-F). In control analyses, no staining was detectable when IgM-positive serum was applied to control-transfected cells or when antiIgG secondary antibodies were applied to IgM-positive serum (see Fig 1B, C), or when transfected cells were incubated with serum/CSF of control patients. Serum of patients with IgG antibodies against NR1/NR2 heterodimers (but not NR1 homodimers) showed identical immunofluorescence patterns on rat brain as serum with antibodies against NR1 alone (not shown).

In a few patients, repeated lumbar puncture was performed to acquire material for further analyses or to monitor white cell counts. Eight follow-up CSF/serum pairs were available for analysis (Fig 3). In 2 cases, increasing antibody titers were observed during the first weeks, suggesting a newly stimulated B cell-mediated response (patients 9, 11; Table 2). Interestingly, the serum antibodies in patient 11 further increased between weeks 7 and 17, whereas the CSF titers declined parallel to clinical improvement. Conversely, detection of high antibody titers at days 4 to 9 in some HSE patients suggests that the NMDAR antibodies already existed at the onset of HSE (patients 1,2). At follow-up obtained $>1$ year 
after symptom presentation, a reduction of antibody titers in serum and CSF was usually observed (eg, patients 3,13).

In many HSE patients (HSV-1 positive) of the present cohort, further neurotropic viruses were excluded. CSF PCR for HSV-2 was performed in all, for VZV in 40, for cytomegalovirus in 21, and for Epstein-Barr virus in 11 of the 44 patients and found negative in all tested samples (not shown). Conversely, no NMDAR antibodies were detected in the serum or CSF of the 20 control patients with PCR-confirmed enterovirus or VZV encephalitis (no serum of VZV cases available; Fig 4).

\section{Characteristics of NMDAR Antibody-Negative versus Antibody-Positive Patients}

The presence of NMDAR autoantibodies in a subgroup of patients raises the question of whether the clinical picture might be different. All patients (except patient 10) responded well to treatment with acyclovir (ie, cessation of seizures, improved level of consciousness, no fever). Basic CSF parameters (white blood cells, red blood cells, protein concentration) were not different between groups (not shown). A higher percentage of female compared to male patients developed antibodies; however, this did not reach statistical significance. Although they were more prevalent in the antibody-positive cohort, we did not find significant differences regarding the presence of epileptic seizures or neuropsychological or psychiatric symptoms during the acute disease phase (1-3 weeks; Table 3). We further detected a difference with longer intervals between first prodromal signs and clinical admission $(p<0.05)$ in the antibody-positive cohort. The clinical course of patients was not different between groups with NMDAR antibodies of the IgG, IgM, or IgA class. No tumors (including teratomas) were reported in any of the patients during the acute encephalitis, but no systematic tumor follow-up was applied in the majority of patients.

\section{Pathogenic Effect of IgM Antibodies}

The pathogenic role of NMDAR antibodies is well established for antibodies of the $\mathrm{IgG}$ class $^{11,15}$ and was recently also demonstrated for the IgA class. ${ }^{14}$ To investigate whether antibodies of the IgM class can downregulate neuronal NMDA receptors in a similar way, we examined the effect of patients' sera using primary hippocampal cell cultures. Neurons incubated with patient serum for 3 days showed a substantial decrease of NMDAR in the membrane fraction (see Fig 2A). This effect was not detectable using serum of healthy controls. Although IgG, IgM, and IgA antibodies show identical effects in neuronal cell culture systems, final proof for the downregulation of NMDAR in vivo is only available for IgG antibodies against the NMDAR. ${ }^{16}$

Similar to the effects previously shown for NMDA-IgA antibodies, ${ }^{14}$ the effect of sera from patients with IgM NMDAR antibodies was not limited to the loss of NMDAR from the cell membrane. In addition, the expression of the synaptic marker synapsin was markedly reduced in primary hippocampal neuron cultures 3 days after serum incubation, whereas incubation with control serum had no effect (see Fig 2B-D). These findings suggest that antibodies of all Ig classes against NMDAR could be pathogenic in patients with HSE.

\section{Discussion}

Our study demonstrates that NMDAR antibodies are frequently present in patients with HSE but not other viral encephalitides, and that these antibodies alter the levels of NMDAR and other synaptic proteins. These findings may have implications for the diagnosis and treatment of some patients with HSE and are potentially relevant for clinical decision making. Shared mechanisms between infectious and autoimmune brain diseases are well known, and coincidence of NMDAR antibodies and HSV positivity per PCR in the CSF of 
encephalitis patients has been observed in single cases, ${ }^{17}$ but not addressed systematically. The present finding of high frequency (30\%) of NMDAR IgM, IgA, or IgG antibodies in patients with encephalitis that is HSV positive by PCR raises the question of how the specific autoimmune antibody response is stimulated in this infectious disease. It might be possible that in NR1-IgG-positive patients, NMDAR antibodies reflect the coincidence of HSE and anti-NMDAR encephalitis. However, this seems unlikely, given that encephalitides are uncommon clinical events and HSE has an incidence of between 1 and 3 cases per million each year. ${ }^{1}$ Also, the absence of the typical multistage clinical picture of NMDAR encephalitis, the age of most patients, and pathological magnetic resonance imaging (MRI) findings strongly argue that this would not account for our patients. Finally, there are no data that patients with classical IgG anti-NMDAR encephalitis developed HSE during or after the acute stage of the disease.

More likely, the virus-induced destruction of neurons may initiate a primary autoimmune response against NMDAR by presentation of tissue that is normally shielded from systemic immunity (immune privilege of the CNS). Alternatively, CNS inflammation in the course of HSE may lead to immunological activation, resulting in a polyspecific B-cell activation, as seen in other types of CNS inflammation such as multiple sclerosis. ${ }^{12}$

The present findings have potential clinical and therapeutic implications. For example, postHSE NMDAR antibodies could explain the choreoathetosis reported in some patients, which occurred during the first months after infection (ie, not directly caused by the virus) and were refractory to acyclovir. ${ }^{8}$ Similarly, NMDAR antibodies after HSE may persist and could explain persistent symptoms, postencephalitic seizures, and relapses after viral clearance has been achieved. ${ }^{7}$ Furthermore, the controversial issue related to the benefit of corticosteroids in some HSE patients ${ }^{5}$ may lead to an explanation in future studies comparing larger cohorts of HSE patients with and without NMDAR antibodies.

We were unable to detect significant clinical differences between the antibody-positive and antibody-negative patient groups, or between groups containing a given Ig class (IgG, IgM, or IgA), during the first days after disease onset. Also, the lack of a long-term follow-up in most patients did not allow conclusions about clinical differences beyond the acute phase of encephalitis. Thus, it is currently unclear whether a difference would be detected with larger sample sizes or longer follow-up. Interestingly, we detected significantly longer intervals between the first prodromal symptoms and hospital admission in patients with NMDAR antibodies. Future studies will determine whether the antibodies are the cause of such symptoms (ie, cognitive deficits rather than headache or fatigue ${ }^{18}$ ), which are also easily (ie, earlier) noted by relatives, but do not result in immediate clinical admission.

The presence of NMDAR antibodies is not equivalent to the diagnosis of anti-NMDAR encephalitis, a distinct disorder associated with the presence of NR1-specific IgG antibodies in the CSF. Compared with large series of patients with anti-NMDAR encephalitis, the patients with NMDAR antibodies identified in the current study were different. Whereas the median age of most patients with anti-NMDAR encephalitis is $\sim 20$ years, and most have normal or mild brain MRI abnormalities, moderate CSF pleocytosis (median white blood cell count of 30 cells $/ \mathrm{mm}^{3}$ ), and normal or mildly increased CSF protein concentration, ${ }^{19,20}$ the patients of this study are older, and have higher levels of CSF pleocytosis (median, 237 cells $/ \mathrm{mm}^{3}$ ) and protein concentration (median, $95 \mathrm{mg} / \mathrm{dl}$ ), suggesting a more intense inflammatory response. Differences related to age, MRI, and CSF findings between antiNMDAR encephalitis and HSE have also been noted by other investigators. ${ }^{21,22}$ Furthermore, almost all patients with anti-NMDAR encephalitis have CSF antibodies, whereas some of the HSE patients were only NMDAR antibody positive in serum. 
Although the presentation and subsequent syndrome related to anti-NMDAR encephalitis is usually different from HSE, our study shows that conversely, some patients positive for HSV by CSF PCR and with clinical, MRI, and CSF features of HSE can have a broad repertoire of NMDAR antibodies. Based on these findings and the demonstration that the antibodies have effects on primary cultures of neurons, we conclude that NMDAR antibodies should be determined in patients with HSE to learn more about the correlation of clinical disease and antibody titers. In addition to the IgG class of antibodies (typical of antiNMDAR encephalitis), antibody screening should include $\operatorname{IgA}$ and IgM, which appear to be more frequent than IgG in patients with HSE. Although the present findings need further evaluation in prospective studies, they suggest that the subgroup of patients with HSE and NMDAR antibodies may benefit from immunotherapy.

\section{Acknowledgments}

This study has been supported by grants from the German Academic Exchange Service to H.P. (DAAD, D/10/ 43923) and by grants from the National Institutes of Health RO1NS077851, RO1MH094741, Fundació la Marató de TV3, and Fondo de Investigaciones Sanitarias (FIS, PI11/01780) to J.D.

\section{References}

1. Steiner I, Kennedy PG, Pachner AR. The neurotropic herpes viruses: herpes simplex and varicellazoster. Lancet Neurol. 2007; 6:1015-1028. [PubMed: 17945155]

2. Whitley, R. Herpes simplex virus. In: Sheld, W.; Whitley, R.; C, Marra, editors. Infections of the central nervous system. Philadelphia, PA: Lippincott Williams \& Wilkins; 2004. p. 123-144.

3. Raschilas F, Wolff M, Delatour F, et al. Outcome of and prognostic factors for herpes simplex encephalitis in adult patients: results of a multicenter study. Clin Infect Dis. 2002; 35:254-260. [PubMed: 12115090]

4. Sellner J, Dvorak F, Zhou Y, et al. Acute and long-term alteration of chemokine mRNA expression after anti-viral and anti-inflammatory treatment in herpes simplex virus encephalitis. Neurosci Lett. 2005; 374:197-202. [PubMed: 15663962]

5. Kamei S, Sekizawa T, Shiota H, et al. Evaluation of combination therapy using aciclovir and corticosteroid in adult patients with herpes simplex virus encephalitis. J Neurol Neurosurg Psychiatry. 2005; 76:1544-1549. [PubMed: 16227548]

6. Meyding-Lamade UK, Oberlinner C, Rau PR, et al. Experimental herpes simplex virus encephalitis: a combination therapy of acyclovir and glucocorticoids reduces long-term magnetic resonance imaging abnormalities. J Neurovirol. 2003; 9:118-125. [PubMed: 12587075]

7. Skoldenberg B, Aurelius E, Hjalmarsson A, et al. Incidence and pathogenesis of clinical relapse after herpes simplex encephalitis in adults. J Neurol. 2006; 253:163-170. [PubMed: 16222428]

8. De Tiege X, Rozenberg F, Des Portes V, et al. Herpes simplex encephalitis relapses in children: differentiation of two neurologic entities. Neurology. 2003; 61:241-243. [PubMed: 12874408]

9. Ropper, A.; Brown, R. Adams and Victor's principles of neurology. 9th ed. New York, NY: McGraw-Hill; 2009.

10. Wandinger KP, Saschenbrecker S, Stoecker W, Dalmau J. Anti-NMDA-receptor encephalitis: a severe, multistage, treatable disorder presenting with psychosis. J Neuroimmunol. 2010; 231:8691. [PubMed: 20951441]

11. Dalmau J, Gleichman AJ, Hughes EG, et al. Anti-NMDA-receptor encephalitis: case series and analysis of the effects of antibodies. Lancet Neurol. 2008; 7:1091-1098. [PubMed: 18851928]

12. Reiber H, Ungefehr S, Jacobi C. The intrathecal, polyspecific and oligoclonal immune response in multiple sclerosis. Mult Scler. 1998; 4:111-117. [PubMed: 9762657]

13. Höltje M, Djalali S, Hofmann F, et al. A 29-amino acid fragment of Clostridium botulinum C3 protein enhances neuronal outgrowth, connectivity, and reinnervation. FASEB J. 2009; 23:11151126. [PubMed: 19047066]

14. Prüss H, Höltje M, Maier N, et al. IgA NMDA receptor antibodies are markers of synaptic immunity in slow cognitive impairment. Neurology. 2012; 78:1743-1753. [PubMed: 22539565] 
15. Prüss H, Dalmau J, Harms L, et al. Retrospective analysis of NMDA receptor antibodies in encephalitis of unknown origin. Neurology. 2010; 75:1735-1739. [PubMed: 21060097]

16. Manto M, Dalmau J, Didelot A, et al. In vivo effects of antibodies from patients with anti-NMDA receptor encephalitis: further evidence of synaptic glutamatergic dysfunction. Orphanet J Rare Dis. 2010; 5:31. [PubMed: 21110857]

17. Davies G, Irani SR, Coltart C, et al. Anti-N-methyl-D-aspartate receptor antibodies: a potentially treatable cause of encephalitis in the intensive care unit. Crit Care Med. 2010; 38:679-682. [PubMed: 20016378]

18. Finke C, Kopp UA, Prüss H, et al. Cognitive deficits following anti-NMDA receptor encephalitis. J Neurol Neurosurg Psychiatry. 2011; 83:195-198. [PubMed: 21933952]

19. Gable MS, Sheriff H, Dalmau J, et al. The frequency of autoimmune N-methyl-D-aspartate receptor encephalitis surpasses that of individual viral etiologies in young individuals enrolled in the California Encephalitis Project. Clin Infect Dis. 2012; 54:899-904. [PubMed: 22281844]

20. Dalmau J, Lancaster E, Martinez-Hernandez E, et al. Clinical experience and laboratory investigations in patients with anti-NMDAR encephalitis. Lancet Neurol. 2011; 10:63-74. [PubMed: 21163445]

21. Gable M, Gavali S, Radner A, et al. Anti-NMDA receptor encephalitis: report of ten cases and comparison with viral encephalitis. Eur J Clin Microbiol Infect Dis. 2009; 28:1421-1429. [PubMed: 19718525]

22. Granerod J, Ambrose HE, Davies NW, et al. Causes of encephalitis and differences in their clinical presentations in England: a multicentre, population-based prospective study. Lancet Infect Dis. 2010; 10:835-844. [PubMed: 20952256] 

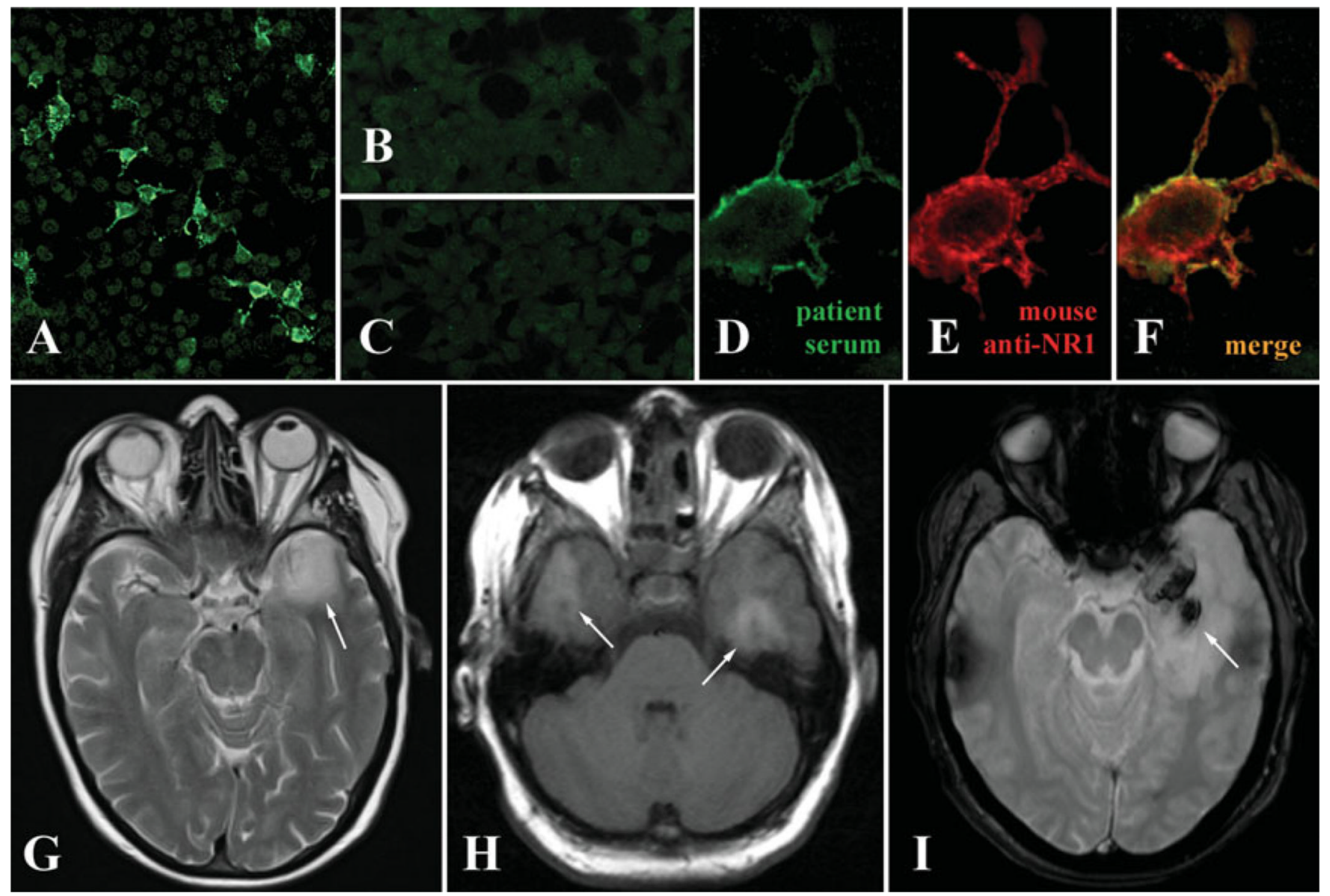

FIGURE 1.

N-Methyl-D-aspartate receptor (NMDAR) autoantibodies in herpes simplex encephalitis (HSE) patients. (A) Immunopositive staining of transfected HEK cells overexpressing the NR1 subunit of NMDARs when probed with patient serum and anti-immunoglobulin (Ig)M secondary antibodies. (B) No staining is observed in control-transfected cells. (C) No staining is observed of transfected cells probed with IgM-positive serum, but an anti-IgG secondary antibody. (D-F) Higher magnification of NR1-transfected cells demonstrating colabeling of patient IgM and a murine anti-NR1 antibody (Biomol International, Plymouth Meeting, PA; dilution, 1:1,000). (G-I) The brain magnetic resonance imaging of patients with HSE $(\mathrm{G})$ can be indistinguishable from imaging in NMDAR encephalitis $(\mathrm{H})$ with predominant affection of the temporal lobes (arrows). However, large hemorrhagic changes in the temporal lobes are typical for HSE (I). [Color figure can be viewed in the online issue, which is available at annalsofneurology.org.] 

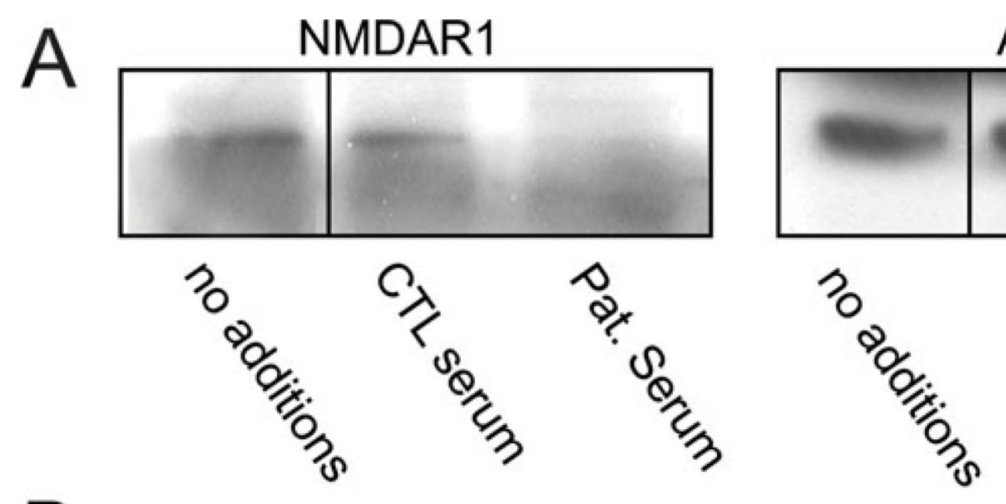

Actin

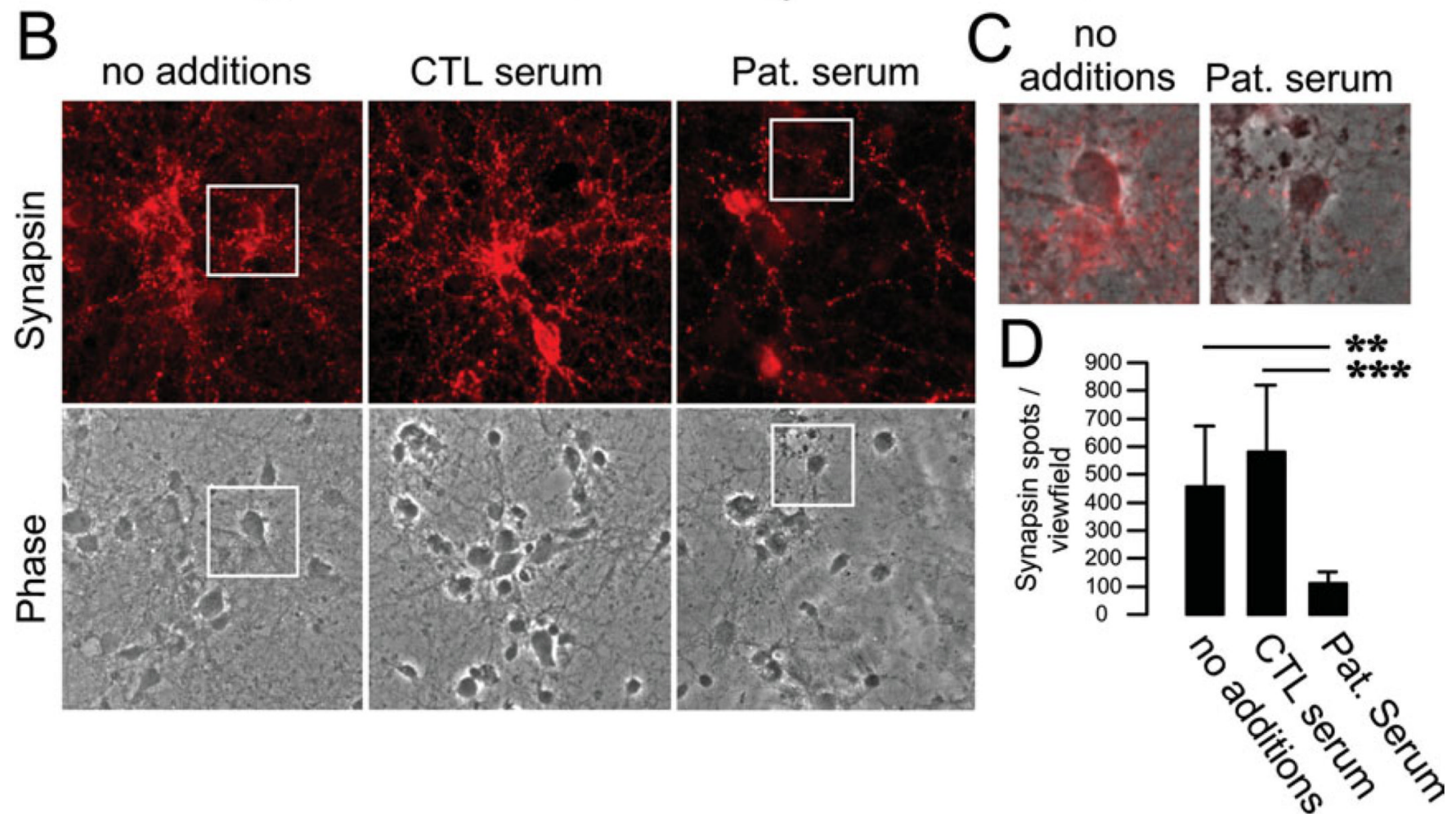

FIGURE 2.

Downregulation of membrane N-methyl-D-aspartate receptors (NMDARs) and synaptic proteins by patient serum containing NMDAR immunoglobulin (Ig)M antibodies. (A) Primary mouse hippocampal neurons were incubated for 3 days with patient serum (1:100 dilution), and the membrane fraction was run in Western blots. Staining against NR1 subunits revealed a strong downregulation of NMDARs following incubation with IgMpositive patient serum (Pat. serum). Incubation with control serum (CTL serum) or media (no additions) had no effect on NMDAR expression. Actin was used for loading control. (B) Immunostaining of hippocampal neurons incubated with patient serum for 3 days led to a dramatic reduction of synapsin-positive clusters. (C) Higher magnification and overlay of the insets in B. (D) Quantification of synapsin spots demonstrating profound downregulation after treatment with NMDAR antibody-positive serum. $* * p<0.005$, *** $p<0.001$. [Color figure can be viewed in the online issue, which is available at annalsofneurology.org.] 

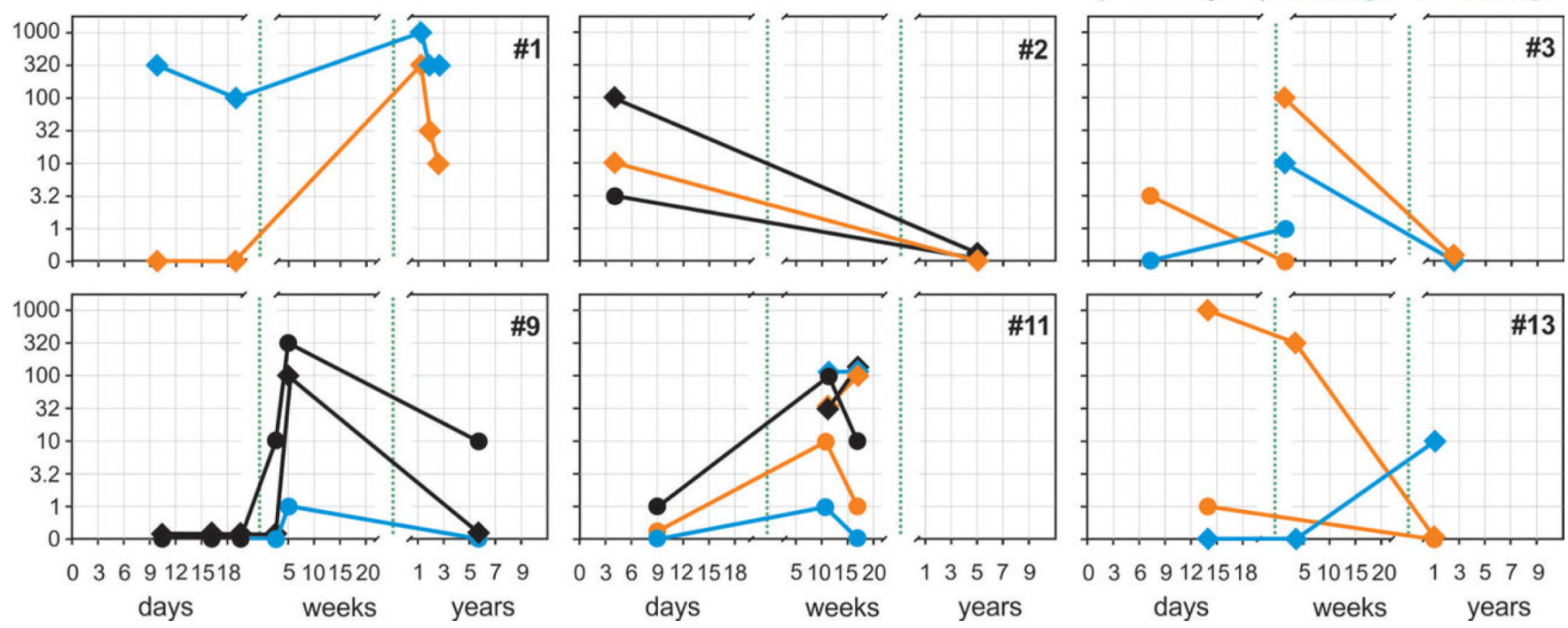

FIGURE 3.

Differential kinetics of N-methyl-o-aspartate receptor (NMDAR) antibody titers of immunoglobulin (Ig)A, IgG, or IgM subtypes in herpes simplex encephalitis patients (see also Table 1). The development of NMDAR antibody titers during the disease course followed a heterogeneous pattern (day $0=$ hospital admission). In most cases, NMDAR antibodies were present already within the first days of initial clinical presentation (eg, patient 2), too early for a primary immune response and indicative of pre-existing antibodies. However, in some cases antibodies did not evolve before the first week (eg, patient 9), suggesting a newly stimulated B cell-mediated response. Late follow-up (after up to 7 years) generally demonstrated reduction in antibody titers in serum and cerebrospinal fluid (CSF; eg, patients 2, 3, 9, 13). [Color figure can be viewed in the online issue, which is available at annalsofneurology.org.] 


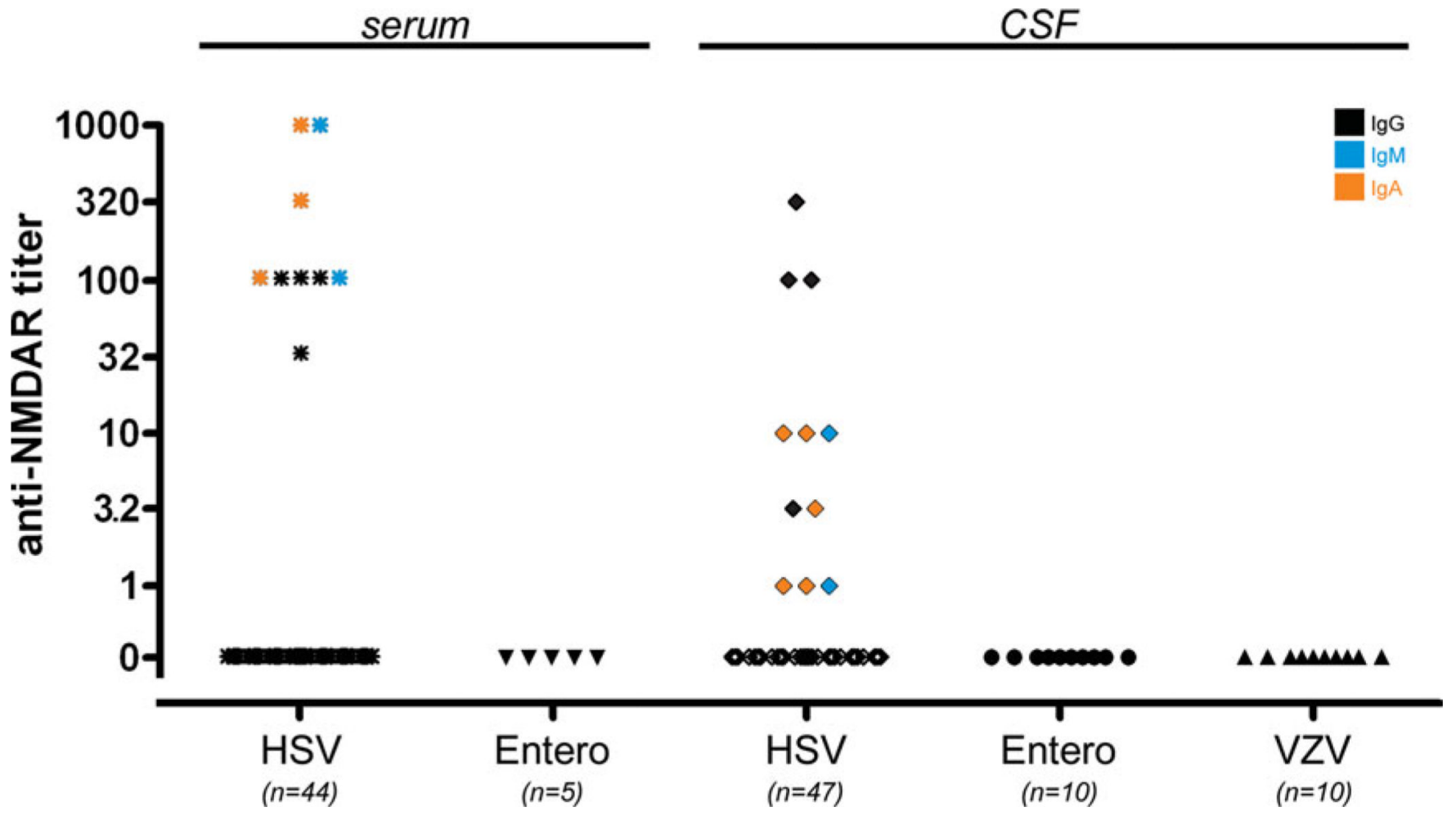

FIGURE 4.

N-Methyl-D-aspartate receptor (NMDAR) antibodies in encephalitis patients. Of patients with herpes simplex virus (HSV) encephalitis, $20.5 \%$ had immunoglobulin $(\operatorname{Ig}) \mathrm{G}$, IgM, or IgA NMDAR antibodies in serum and $23.4 \%$ in cerebrospinal fluid (CSF), whereas no antibodies were detected in the CSF and serum of patients with enterovirus encephalitis or CSF of varicella zoster virus (VZV) encephalitis (serum of VZV cases not available). [Color figure can be viewed in the online issue, which is available at annalsofneurology.org.] 


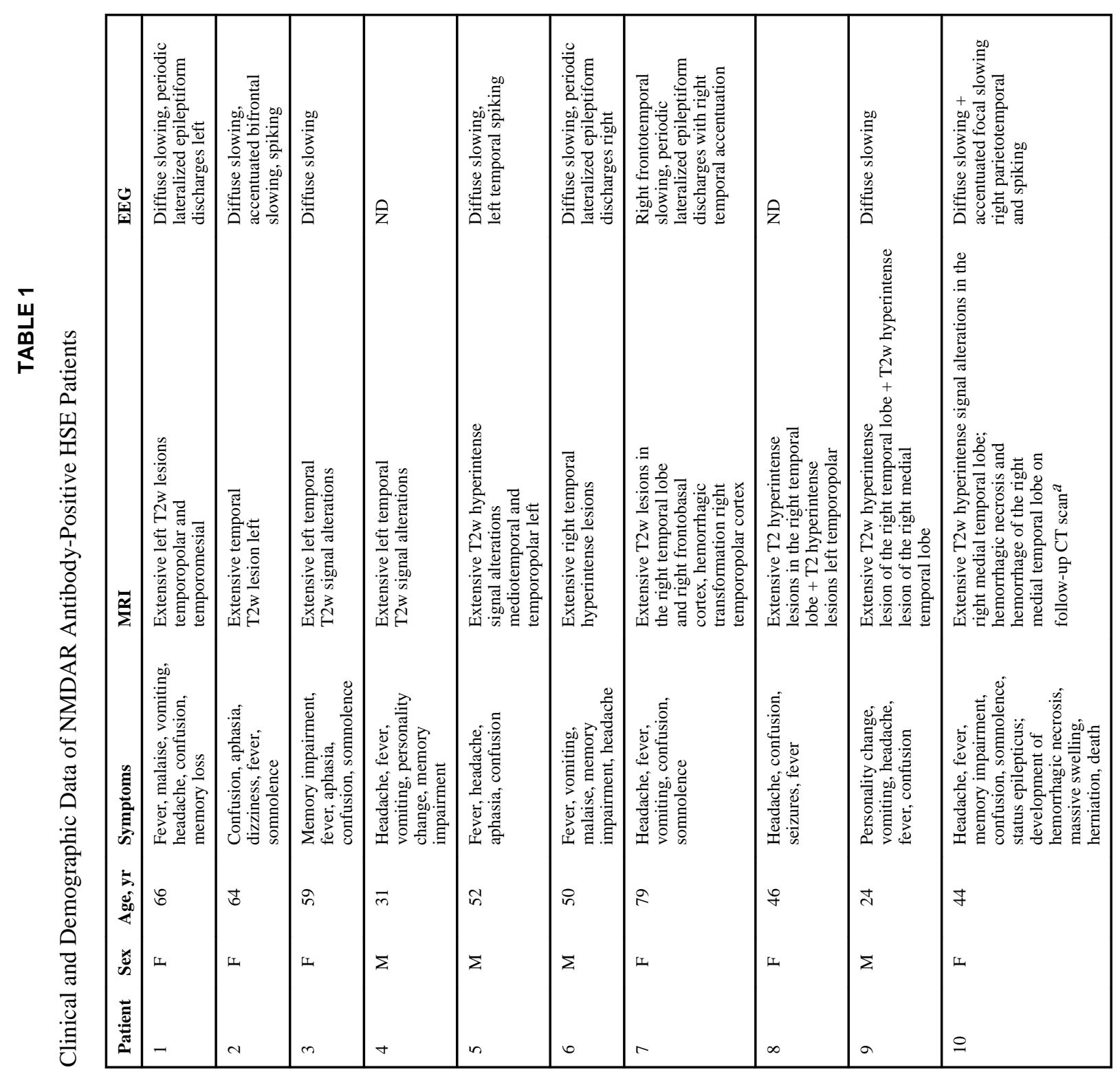

Ann Neurol. Author manuscript; available in PMC 2013 July 29. 


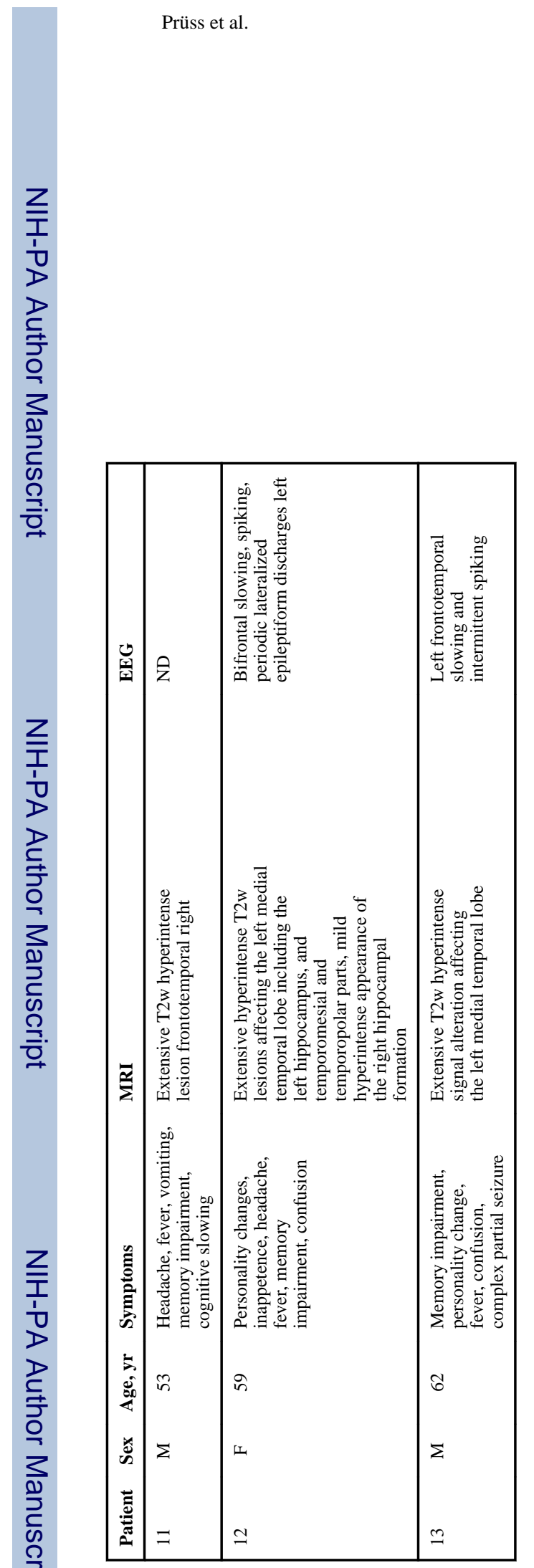

Page 14

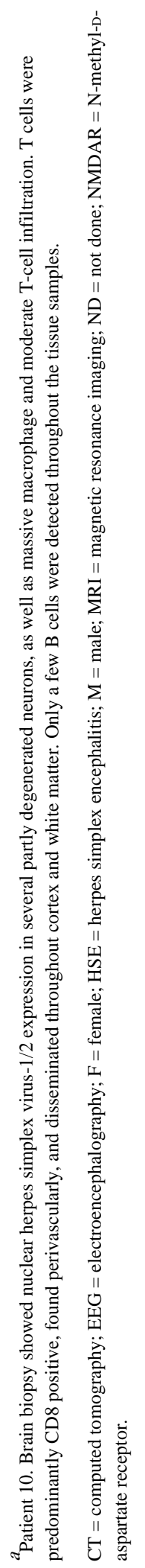

Ann Neurol. Author manuscript; available in PMC 2013 July 29. 


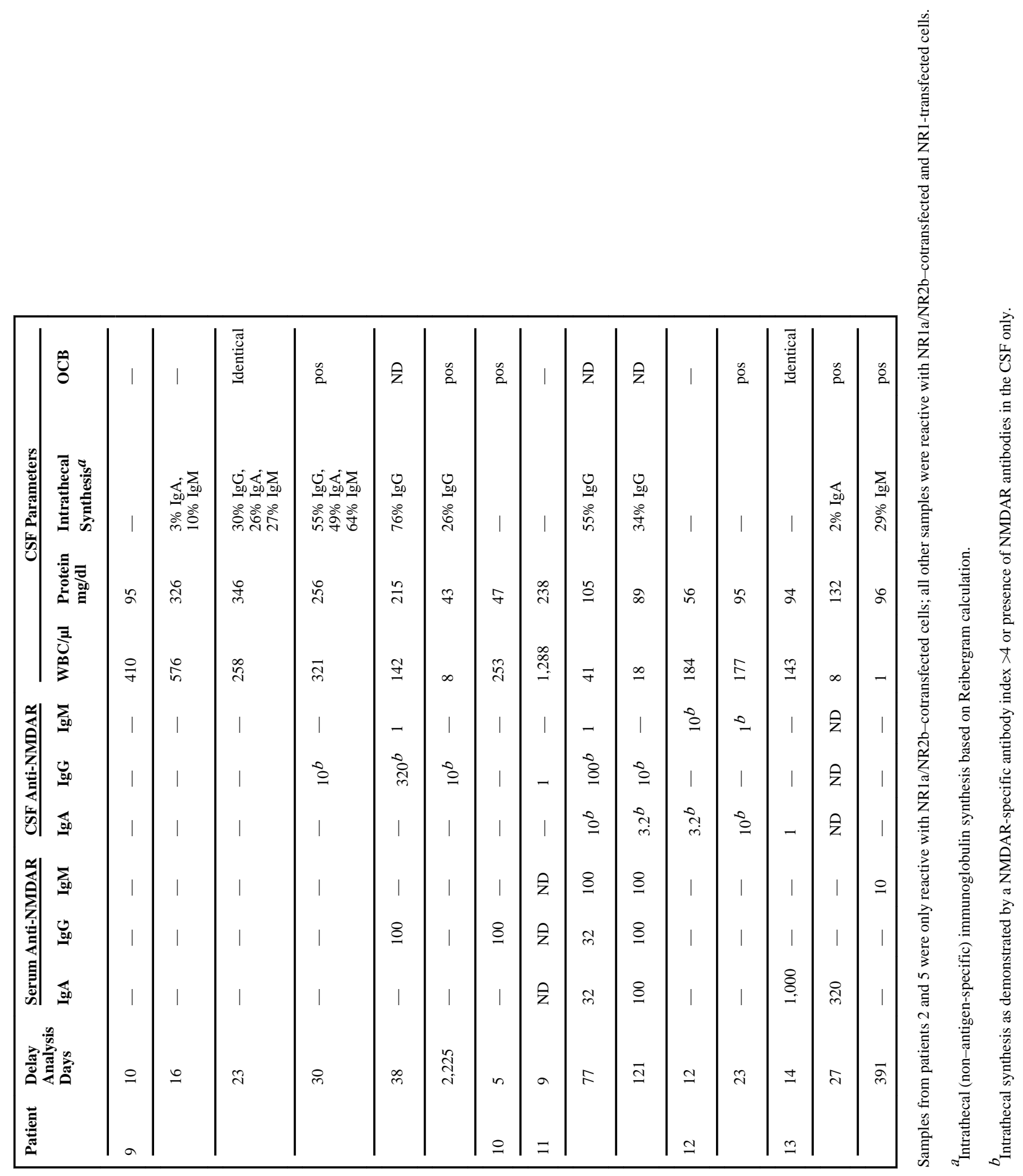


TABLE 3

Clinical Signs and Demographic Features in NMDAR Antibody-Positive versus Antibody-Negative HSE Patients

\begin{tabular}{|c|c|c|c|}
\hline \multirow[t]{2}{*}{ Sign/Feature } & \multicolumn{2}{|c|}{ NMDAR Antibodies } & \multirow[t]{2}{*}{ Probability } \\
\hline & Positive & Negative & \\
\hline $\begin{array}{l}\text { Days between first clinical } \\
\text { signs and admission }\end{array}$ & $6.3 \pm 2.8$ & $3.8 \pm 2.2$ & $p<0.05$, Mann-Whitney $U$ test \\
\hline $\begin{array}{l}\text { Neuropsychiatric/ } \\
\text { neuropsychological symptoms }{ }^{a}\end{array}$ & $3 / 8(38 \%)$ & $5 / 17(29 \%)$ & $\begin{array}{l}\text { NS, chi-square }=0.09, \\
p=0.76, \text { chi-square test }\end{array}$ \\
\hline Epileptic seizures & $2 / 8(25 \%)$ & $2 / 17(18 \%)$ & $\begin{array}{l}\text { NS, chi-square }=0.49, \\
p=0.48, \text { chi-square test }\end{array}$ \\
\hline Gender & $\begin{array}{l}\text { M 6/27 (25\%), } \\
\text { F 7/17 (41\%) }\end{array}$ & $\begin{array}{l}\text { M 21/27 (75\%), } \\
\text { F } 10 / 17(59 \%)\end{array}$ & $\begin{array}{l}\mathrm{NS}, \text { chi-square }=1.80, \\
p=0.18, \text { chi-square test }\end{array}$ \\
\hline Age, yr & $53.0 \pm 14.2$ & $52.6 \pm 17.5$ & NS, $p=0.84$, Mann-Whitney $U$ test \\
\hline
\end{tabular}

${ }^{a}$ For example, confusion, amnestic deficits, behavioral changes.

$\mathrm{F}=$ female $; \mathrm{HSE}=$ herpes simplex encephalitis $; \mathrm{M}=$ male; $\mathrm{NMDAR}=\mathrm{N}$-methyl-D-aspartate receptor; NS = not significant. 